\title{
The halting of contact lines in slender viscous films driven by gravity and surface tension gradients
}

\author{
J. M. Foster \& A. D. Fitt
}

August 31, 2016

\begin{abstract}
The evolution of thin layers of viscous fluid with compact support is considered in a case where the driving forces are gravity and surface tension gradients (which we initially take to be locally constant). In particular, we examine cases where the contact line may initially advance, but then halts at a finite time. Although this phenomenon of halting contact lines is well known, it appears that there was previously little analytical insight into how this occurs. The approach taken here is to seek self-similar solutions local to both the contact line and the halting time. The analysis is split into two parts, namely before and after the halting time. By invoking continuity across $t=0$ (the halting time) it is possible to give a complete asymptotic description of both the advancing and halting processes. It is further shown that the analysis may be extended to encompass various cases where the surface tension gradient is not constant at the contact line. Finally, details are given of some numerical experiments that act as plausibility tests for the results that have been obtained.
\end{abstract}

\section{Introduction}

Marangoni effects (traditionally reported to have first been observed in "tears of wine", see Thomson[1]) arise when flow is generated by gradients of the surface tension $\sigma$. Such gradients are a consequence of the chemical composition and/or the temperature distribution at an interface between two fluids. Though pressure jumps across a flow interface can be maintained by normal stress jumps due to surface tension, tangential surface stresses caused by surface tension gradients must necessarily be sustained by viscous stresses that inevitably lead to fluid motion. Thus a scenario in which the fluid velocity is uniformly zero cannot occur when surface tension gradients are present.

In Pearson's[2] pioneering study surface tension gradients were used to explain the cellular patterns observed in drying paint. Since then, many authors have considered a variety of different scenarios in which surface tension gradients 
drive instabilities throughout a viscous film. Giving an exhaustive list (or even a summary) of all the extant work on flows with surface tension gradients would be almost impossible. Instead, we direct the interested reader towards either the review of Oron et. al.[3], or to one of the many books[4, 5] now available on Marangoni effects.

One avenue of previous study, which is relevant to the work presented here, concerns the spreading of thin viscous regions with compact support under various forces, see either Myers'[6] review or the large body of work contributed by Huppert[7]. The contact lines of such regions can exhibit a wealth of interesting different behaviours and asymptotic properties depending on the kind of forces that act and their relative importance. Many kinds of evolution are possible: contact lines that remain stationary for a finite time and then begin to move have been identified by Aronson et. al.[8] and Lacey et. al.[10, 9]. These 'waiting time' solutions were initially thought to be governed solely by the structure of the solution local to the contact line. However, these locally determined solutions were later shown to be sub-cases of a much broader family of solutions that are governed by the global behaviour. When absorption competes with gravity, previous studies[11, 12, 13, 14] have shown that contact lines may move with either finite or infinite speed, and may or may not suffer finite-time extinction, see Ferreira \& Vazquez[15] and Grundy[16]. In addition, solutions can, under the correct conditions, exhibit a 'reversing' contact line where the compact support of the solution initially expands and then subsequently contracts. In Foster at. al.[17] the asymptotic structure of these 'reversing' solutions have been elucidated using self-similar reductions local to the contact line and local to the 'reversing time'.

Several authors $[18,19,20,21]$ have also given analytical descriptions of the rupture of thin films. When van der Waals forces act, rupture can be described by a family of local self-similar solutions. Numerical simulations of the timedependent governing PDE have indicated that only one of these self-similar solutions is observed in practice. A related problem, in more spatial dimensions, has been considered by Yatim et. al.[22, 23] who examined the evolution of rivulets/dry patches using similarity reductions, asymptotic analysis and shooting techniques.

In addition to these analytical insights an appreciable number of previous studies $[24,25,26]$ have considered how to evolve contact lines in such problems using wholly numerical methods. It transpires that these are particularly difficult problems to tackle numerically owing to the shear stress singularity that exists at any contact line.

In the current study we wish to show that a different phenomenon may occur when surface tension gradients compete with gravity. In particular, flows may exist where an advancing contact line halts at a finite time, after which the fluid-air interface continues to evolve while the position of the contact line remains fixed. We consider this scenario in one-spatial dimension and will give an analytical description of the local halting process by splitting the analysis into two parts - one part for the behaviour prior to the halting time $(t<0)$, and the other for the behaviour after the halting time $(t>0)$. In each case, a 
self-similar reduction will be employed to derive an ODE for the evolution of the solution. It will transpire that neither of these ODEs admit any suitable exact solutions so we proceed by examining the asymptotic behaviour of the solutions to each of the ODEs near the singular points. We then develop a numerical shooting scheme, which makes use of the aforementioned asymptotic behaviours, to compute solutions to each of the ODE problems. Finally, we impose continuity of the solution across $t=0$ to derive physically meaningful solutions to the governing PDE local to the contact line and local to the halting time.

\subsection{Equations of Motion}

Here we briefly summarise the derivation of suitable equations and boundary conditions (a more detailed version of this derivation is given in Howison et. al. [27]). We assume that a thin two-dimensional region $\Sigma$ of an incompressible Newtonian viscous fluid with compact support moves in air on a solid flat substrate (at $z=0)$. The fluid-air interface, $z=h(x, t)$, of $\Sigma$ moves as a result of viscous forces, gravity and surface tension gradients. The fluid equations of motion are the Navier-Stokes equations and the condition of incompressibility. Appropriate boundary conditions are: (i) no slip on the substrate; (ii) the kinematic condition on the fluid-air interface; (iii) dynamic conditions on the fluid-air interface that are derived by carrying out a standard force balance on $z=h(x, t)$.

The equations and boundary conditions may now be examined in the appropriate thin layer limit. Denoting non-dimensional quantities with an overbar, we set $t=(L / U) \bar{t}, x=L \bar{x}, z=h_{0} \bar{z} u=U \bar{u}, w=\left(U h_{0} / L\right) \bar{w}, h=h_{0} \bar{h}$ and $p=\left(\mu U L / h_{0}^{2}\right) \bar{p}$ where $h_{0}$ and $L$ respectively denote a typical height and lateral extent of $\Sigma$ and $U$ a typical flow speed. If the drop is thin so that $\epsilon=h_{0} / L \ll 1$ and also $\epsilon^{2} L U / \nu \ll 1$, then we find that so long as $g L^{2} \epsilon^{3} /(U \nu)=O(1)$ the leading order (dimensional) equations may be solved to give

$$
p=\rho g(h-z)-\sigma h_{x x}, \quad h_{t}=\left(\frac{h^{3} p_{x}}{3 \mu}\right)_{x}-\left(\frac{h^{2} \sigma_{x}}{2 \mu}\right)_{x} .
$$

In this study we wish to consider the specific case where $\sigma$ may be written $\sigma=\sigma_{0}+\sigma_{1}(x) \Delta \sigma$, and the relative sizes of the Marangoni and capillary numbers $M$ and $C$ respectively are

$$
M=\frac{\epsilon \Delta \sigma}{\mu U}=O(1), \quad C=\frac{\epsilon^{3} \sigma_{0}}{\mu U} \ll 1 .
$$

The equation of motion for the fluid-air interface, $z=h(x, t)$, is therefore given by

$$
h_{t}=\left(\frac{\rho g}{3 \mu}\right)\left(h^{3} h_{x}\right)_{x}-\frac{1}{2 \mu}\left(\sigma_{x} h^{2}\right)_{x} .
$$

The governing equation (3) must be closed by supplying boundary and initial conditions. We assume that at any contact line $x=s(t)$ where $h$ becomes equal 
to zero the flux through the contact line is zero (see Foster et al.[17] for details). Thus

$$
h(s(t), t)=0, \quad \frac{d s}{d t}=\left.\left(-\frac{\rho g h^{2}}{3 \mu} \frac{\partial h}{\partial x}+\frac{\sigma_{x} h}{2 \mu}\right)\right|_{x=s(t)} .
$$

In this study we will also assume (mainly for simplicity) that $h_{x}(0, t)=0$ and deal with a single contact line. One consequence of this condition is that the total film volume in $x>0$ is not necessarily conserved. Many alternative conditions are possible, but the choice is not important as it will be the analysis local to the contact line that is of most interest. We further specify the initial film height and position of the contact line so that the initial conditions are

$$
h(x, 0)=h_{0}(x), \quad s(0)=s_{0}
$$

where $h_{0}(x)$ has compact support.

The quantities $h, s, x$ and $t$ may now be scaled to remove some of the physical parameters from the problem. It is straightforward to show that without loss of generality the two ratios of physical parameters in the governing equation may be scaled away, and $s_{0}$ may be scaled to unity. The only variables that affect the solutions are therefore the initial data $h_{0}(x)$ and the nature of the surface tension gradient $\sigma_{x}$. In many problems of practical interest the surface tension gradient must be determined as part of the solution to the problem. In some cases (e.g. if the substrate is held at a prescribed temperature and the fluid has appropriate thermal properties) however it is possible to determine the behaviour of $\sigma_{x}$ (at least locally to the contact line) apriori. In the remainder of this study we shall assume that this is the case. The problem therefore becomes

$$
\frac{\partial h}{\partial t}=\frac{\partial}{\partial x}\left(h^{3} \frac{\partial h}{\partial x}-\sigma_{x} h^{2}\right)
$$

with

$$
\begin{gathered}
h(x, 0)=h_{0}(x), \quad s(0)=1, \\
h_{x}(0, t)=0, \quad h(s(t), t)=0, \quad \frac{d s}{d t}=-h^{2} \frac{\partial h}{\partial x}+\left.\sigma_{x} h\right|_{x=s(t)} .
\end{gathered}
$$

For convenience, the final problem that we shall henceforth consider will be slightly different: we assume (by relabelling the coordinates and/or time if required) that the left contact line of the solution is at position $x=s(t)$ and moves leftwards for $t<0$, then at time $t=0, s(0)=0$ and the contact line halts and henceforth remains stationary for $t>0$. Analogous conditions may be added if additional contact lines are included, but we do not pursue this further here. We note that in the case $\sigma_{x}=0$, equation (6) reduces to a non-linear diffusion equation describing the evolution of a thin viscous film spreading on a flat solid substrate under the influence of gravity alone. It is well known that in this case the contact line of the solution advances for all time and never halts.

The analysis will now proceed to give the asymptotic details of a single left contact line that moves leftwards for a finite time, before halting. After the halting time the solution evolves further but the left contact line remains 
stationary. We shall examine suitable self-similar reductions for both $t<0$ and $t>0$ that give rise to two ODE problems that must be solved. Imposing the condition of continuity across $t=0$ closes the problem and unique solutions to each of the ODE problems will then be obtainable via a numerical shooting scheme. We shall then consider several additional forms that the Marangoni forces can take where the surface tension gradient is not locally constant. Some numerical experiments will also be performed to illustrate the plausibility of some of the results.

\section{The case $\sigma_{x} \sim 1$ as $h \rightarrow 0^{+}$}

We begin by studying the case where the surface tension gradient is constant (which, without loss of generality, we take to be one) near to the contact line, i.e. we take $\sigma_{x} \sim 1$ as $h \rightarrow 0^{+}$. The governing equation and boundary conditions local to the left contact line can be reduced to

$$
\frac{\partial h}{\partial t}=\frac{\partial}{\partial x}\left(h^{3} \frac{\partial h}{\partial x}-h^{2}\right), \quad h(s(t), t)=0, \quad \dot{s}=-h^{2} \frac{\partial h}{\partial x}+\left.h\right|_{x=s(t)},
$$

(in this case the local problem, which may be formally derived from (6)-(8) by scaling $h, t$ and $x$ by $\epsilon, \epsilon$ and $\epsilon^{2}$ respectively, is essentially the same as the full problem, provided suitable matching conditions are specified).

Examining the travelling-wave behaviour of (9) is straightforward: a balance between the time derivative term and the first term on the right-hand side (RHS) of the PDE in (9) yields a possible travelling wave solution of the form

$$
h \sim(-3 \dot{s})^{1 / 3}(x-s(t))^{1 / 3} \text { as } x \rightarrow s(t)^{+} .
$$

This is a wave driven by gravity that pushes the left contact line leftwards. Further, a balance between the time derivative and the second term on the RHS of the PDE in (9) yields a solution local to a left contact line of the form

$$
h \sim \frac{x}{2 t} \quad \text { as } \quad x \rightarrow 0^{+} .
$$

We anticipate that this local solution (with $\dot{s}=0$ ) exists after the contact line has halted and the surface tension gradients draw fluid away from the contact line. Finally, we note that there is an exact solution given by

$$
h=2^{1 / 2} x^{1 / 2} .
$$

The solution (12) does not interest us further in this study as it does not involve a moving contact line. For reasons that will become clear we now discuss the solution first for $t>0$.

\subsection{Behaviour after the halting time}

To examine the asymptotic behaviour of solutions when $t>0$ and the contact line has halted, we employ a similarity reduction of the form

$$
h(x, t)=t H(\phi) \quad \text { with } \quad \phi=\frac{x}{t^{2}} \quad \text { and } \quad s(t)=0,
$$


that reduces (9) to

$$
H-2 H^{\prime} \phi=\left(H^{3} H^{\prime}-H^{2}\right)^{\prime} \quad \text { with } \quad H(0)=0 .
$$

The similarity reduction (13) was originally reported by Gandarias[28] and was subsequently used by Foster et. al.[17] to examine the behaviour of contact lines in a problem where absorption effects are present.

The problem (14) readily admits one non-trivial exact solution, $H=2^{1 / 2} \phi^{1 / 2}$, but this corresponds to the steady solution (12) and is therefore not examined further. Since no other suitable exact solutions appear to exist, we examine the asymptotic behaviour of $H$ near the singular points of (14), i.e. as $\phi \rightarrow 0^{+}$and as $\phi \rightarrow+\infty$. In the near-field we find that

$$
H \sim \frac{1}{2} \phi+\left(A_{1}-\frac{3}{16} \log \phi\right) \phi^{2}+O\left(\phi^{3} \log ^{2} \phi\right) \quad \text { as } \quad \phi \rightarrow 0^{+},
$$

for some as yet undetermined constant $A_{1}$. We note that the near-field behaviour (15) corresponds to the local solution (11). In the far-field we find that

$$
H \sim A_{2} \phi^{1 / 2}+\frac{A_{2}^{2}\left(A_{2}^{2}-2\right)}{2}+O\left(\phi^{-1 / 2}\right) \quad \text { as } \quad \phi \rightarrow+\infty,
$$

where $A_{2}$ is some as yet undetermined constant. Next, we proceed to examine the asymptotic behaviours of the local self-similar solutions for $t<0$.

\subsection{Behaviour prior to the halting time}

To examine the asymptotic behaviour of solutions when $t<0$ and the contact line is advancing, we make use of a similarity reduction of the form

$$
h(x, t)=-t H(\phi) \quad \text { with } \quad \phi=\frac{x}{(-t)^{2}} \quad \text { and } \quad s(-t)=\frac{\Phi}{(-t)^{2}},
$$

where $\Phi$ is an as yet undetermined constant. The local problem may be written as

$$
2 H^{\prime} \phi-H=\left(H^{3} H^{\prime}-H^{2}\right)^{\prime} \quad \text { with } \quad H(\Phi)=0,
$$

which readily admits one non-trivial exact solution, namely $H=2^{1 / 2} \phi^{1 / 2}$. For the reasons discussed in section 2.1 we do not examine this solution further. Instead, we again examine the asymptotic behaviour of $H$ near the singular points of (18), i.e. as $\phi \rightarrow \Phi^{+}$and as $\phi \rightarrow+\infty$. We find that in the near-field:

$$
H \sim(6 \Phi)^{1 / 3}(\phi-\Phi)^{1 / 3}+\frac{6^{2 / 3}}{8 \Phi^{1 / 3}}(\phi-\Phi)^{2 / 3}+O(\phi-\Phi) \quad \text { as } \quad \phi \rightarrow \Phi^{+},
$$

whereas, in the far-field we find that:

$$
H \sim B \phi^{1 / 2}+\frac{B^{2}\left(2-B^{2}\right)}{2}+O\left(\phi^{-1 / 2}\right) \quad \text { as } \quad \phi \rightarrow+\infty,
$$


where $B$ is an as yet undetermined constant. We note that there is a second possible asymptotic behaviour for solutions to (18) in the far-field, namely

$$
H \sim(3 / 10)^{1 / 3} \phi^{2 / 3}+O\left(\phi^{1 / 3}\right) .
$$

However, bearing in mind that the far-field behaviours to (14) and (18) must be identical - in order to ensure continuity of $h(x, t)$ across $t=0$ - one would expect that the behaviour (21) is not manifest during the halting process. To verify that this is the case, we have examined numerical solutions to both the local self-similar problem (18) and the full PDE problem (9). In each case we were unable to observe the behaviour (21) and we therefore do not examine it further here.

With the asymptotic behaviour of the solutions to both the $t<0$ and $t>0$ problems (i.e. (14) and (18)) determined we now discuss a numerical shooting scheme that can be used to (i) join the behaviours (19) and (20) and (ii) join the behaviours (15) and (16). By doing so we will be able to compute physically meaningful halting solutions to (9) that are suitably continuous across $t=0$.

\subsection{The shooting scheme}

Having examined the asymptotic behaviours of solutions to (14) and (18) in both the near- and far-fields we now discuss the details of a numerical shooting scheme that will allow us to find appropriate solutions to (14), (18) and hence (9) and its local boundary conditions. Similar shooting techniques have been used previously: Tuck \& Schwartz[29] used related techniques to begin numerical integration at 'infinity' in a problem that describes the draining of a film down a vertical wall; in addition, Zhang \& Lister[21] employed similar techniques to begin numerical integration near a coordinate singularity of a fourth-order thin film equation. First we consider the solution for $t<0$ before the contact line halts:

Prior to the halting time. The solution for $t<0$ may, in principle, be determined in two different ways; (i) by shooting from $\phi=\Phi$ towards the farfield and adjusting $\Phi$ in (19) as a shooting parameter, or (ii) by shooting from the far-field towards $\phi=\Phi$ and adjusting $B$ in (20) as a shooting parameter. In method (i) one must circumvent the complications associated with integrating away from a singular point - note that along the line $H=0$, the second derivative, $H^{\prime \prime}$, is not well defined. One method for overcoming this difficulty is to choose a $\delta \ll 1$ and use the asymptotic behaviour (19) to fix $H(\Phi+\delta)$ and $H^{\prime}(\Phi+\delta)$ to begin the integration. However, herein lies another problem. Taking a value of $\delta$ that is too small would result in a large numerical error arising from the large value of $H^{\prime}(\Phi+\delta)$. On the other hand, taking a value of $\delta$ that is too large would result in inaccuracy of the asymptotic series (19) and hence inaccuracy of the initial data. To verify that a suitable value of $\delta$ had been chosen we varied $\delta$ (typically through the range $10^{-12}-10^{-4}$ ) and checked

that there were no appreciable changes in the determined value of the shooting 
parameter $\Phi$. In method (ii) similar issues are encountered, in that, one must make a suitable numerical approximation of 'infinity'. However, in practice, we found this approach more time consuming, and, it did not offer any advantages over the more straightforward method (i).

After the halting time. With the solution for $t<0$ determined the condition of continuity of $h(x, t)$ across $t=0$ is enforced to fix $A_{2}=B$. The most straightforward approach for determining the solution for $t>0$ is to shoot from near $\phi=0$ towards the far-field and adjust $A_{1}$ in (15) as a shooting parameter with the aim of reaching the far-field behaviour (16). An analogous technique (to that described above) was used to step away from the singular point, $H=0$, and the same numerical checks were carried out to ensure that a suitable step size, $\delta$, had been chosen.

\subsection{Results}

In this section we employ the numerical scheme described above in section 2.3 to find solutions to (14), (18) and hence (9). Implementing the ODE45[30] routine in MATLAB the following values were determined:

$$
\Phi \approx 0.013, \quad B=A_{2} \approx 1.403, \quad \text { and } \quad A_{1} \approx 10.294
$$

An absolute error tolerance of $10^{-10}$ was used: as the absolute error tolerance was reduced below $10^{-10}$ there were no appreciable changes to the computed solutions. Plots of the results of the numerical computations are shown in figures 1 and 2 for $t<0$ and $t>0$ respectively. Figure 3 has been included to show that the condition of continuity across $t=0$ has been satisfied. Other numerical computations were carried out for a large range of values of the shooting parameters. It was found in all cases that the deviation from the required behaviour was monotone in the shooting parameter. Although far from a rigorous proof, the numerical evidence strongly suggests that the above values of $\Phi, B, A_{1}$ and $A_{2}$ are unique. Figure 4 has been included to show the evolution of $h(x, t)$ and the position of the contact line during this evolution.

\section{$3 \quad$ The case $\sigma_{x} \sim(x-s(t))^{a}$ as $h \rightarrow 0^{+}$}

In this section we will show how the analysis in section 2 can be extended to cases where the surface tension gradient is not constant near the contact line. We shall examine the particular case where $\sigma_{x} \sim(x-s(t))^{a}$ as $h \rightarrow 0^{+}$. In this case the governing PDE and boundary conditions local to the contact line (having scaled away any extraneous coefficients) are

$$
\frac{\partial h}{\partial t}=\frac{\partial}{\partial x}\left(h^{3} \frac{\partial h}{\partial x}-(x-s(t))^{a} h^{2}\right), \quad h(s(t), t)=0, \quad \dot{s}=-h^{2} \frac{\partial h}{\partial x}+\left.(x-s(t))^{a} h\right|_{x=s(t)} .
$$



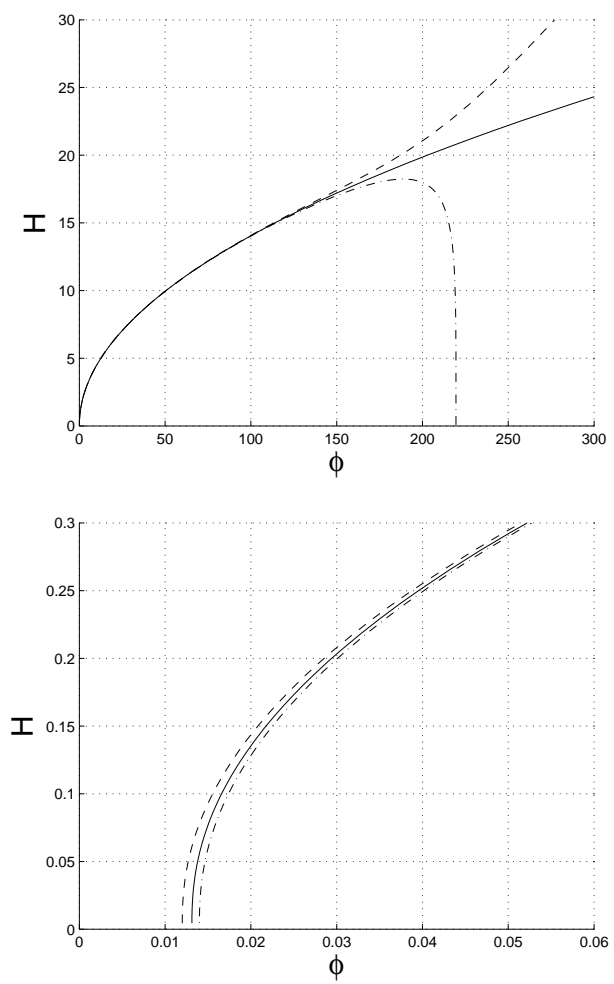

Figure 1: The left-hand panel shows some solutions to (18) emanating from (19). The solid curve shows the solution to (18) emanating from $\Phi \approx 0.013$, whilst the dashed and the dash-dotted curves emanate from values of $\Phi<0.013$ and $>0.013$, respectively. The right-hand panel shows the same solutions close to $\phi=\Phi$ so that the reader can see the important but subtle behaviour in this region. Further numerical experiments were carried out and it was found that for all values of $\Phi<0.013$ the solution curves deviate from the requisite far-field behaviour tending towards positive infinity. Meanwhile, if a value of $\Phi>0.013$ was chosen the solution curve would deviate from the requisite farfield behaviour and tend towards negative infinity. Thus, it is anticipated that there is one unique value of $\Phi(\approx 0.013)$ that leads to the required far-field behaviour.

It may easily be shown that there are two possible types of travelling wave solution, provided that

$$
-\frac{1}{3}<a<\frac{1}{3} .
$$

The lower bound on $a$ ensures that the surface tension gradients are sufficiently weak to allow an advancing contact line to exist at some time during the evolution. We anticipate that when $a \leq-1 / 3$ it is not possible to observe an 

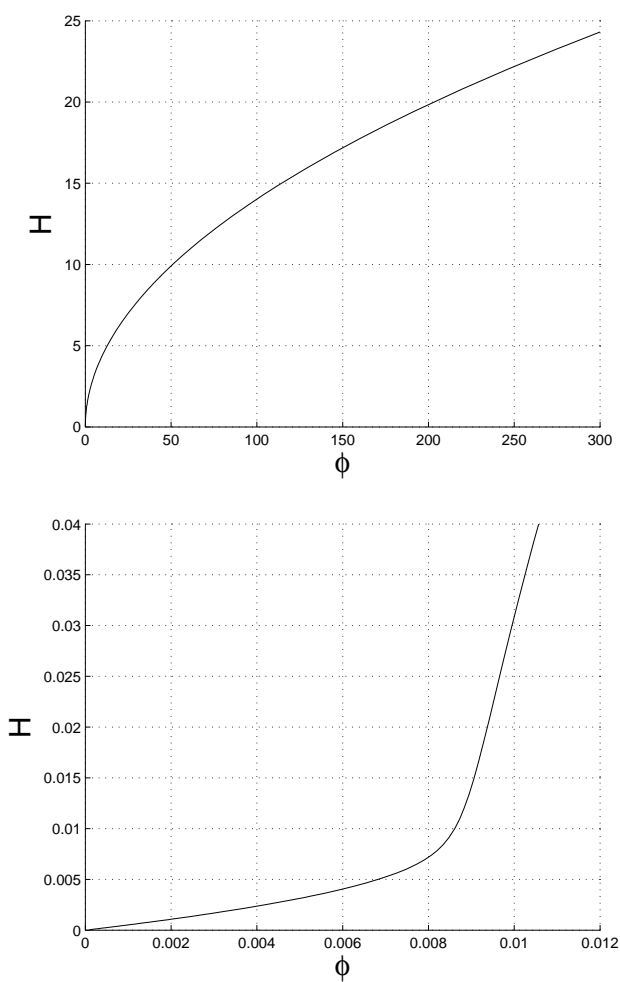

Figure 2: The left-hand panel shows the solution to (14) emanating from (15) with a value of $A_{1} \approx 10.294$ and reaching the requisite far-field behaviour. The right-hand panel shows the same solution close to the origin so that the reader can see the important but subtle behaviour in this region.

advancing contact line and the nature of the solution(s) may be radically different to those that we consider here. The upper bound on $a$ ensures that the qualitative features of the solution resemble the case $\sigma_{x} \sim 1$ as $h \rightarrow 0^{+}$and a physically plausible solution exists for $t>0$. We conjecture (but have not proved) that if $a \geq 1 / 3$ then the surface tension gradients near the contact line are not sufficiently strong to ever stop the contact line. In the parameter regime of interest we observe two different types of solution: The first is a travelling wave due to a balance between the term on the LHS and the first term on the RHS of (23) and takes the familiar form

$$
h \sim(-3 \dot{s})^{1 / 3}(x-s(t))^{1 / 3} .
$$

This is an advancing contact line that is driven by gravity. A second (similarity) solution arises when $s(t)=0$ and there is a balance between the term on the 

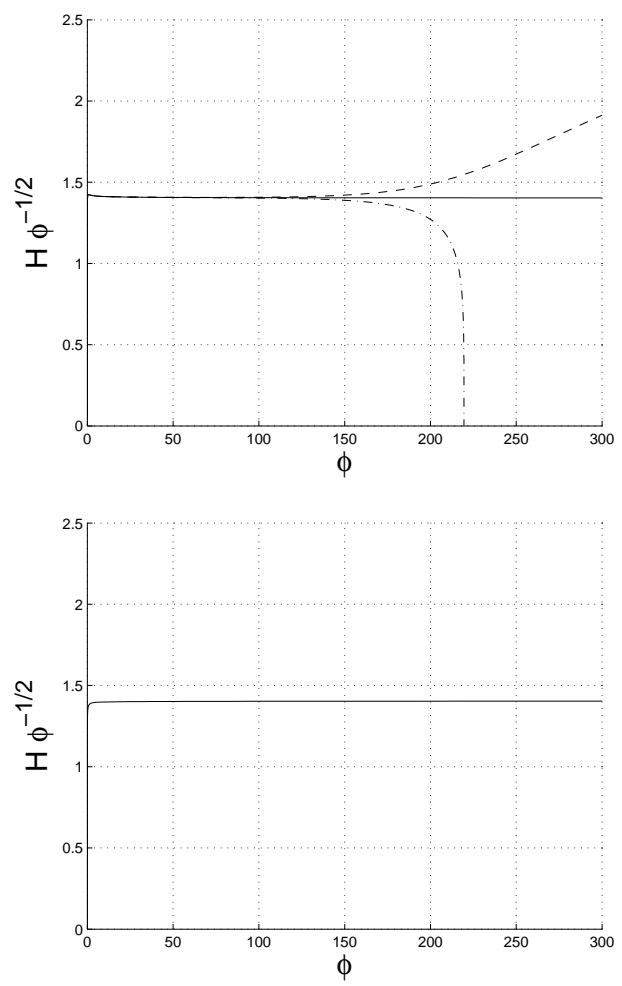

Figure 3: The left-hand panel shows the solution curves from figure 1 and the right-hand panel shows the solution curve from figure 2. By plotting the quantity $H \phi^{-1 / 2}$ against $\phi$ it is straightforward to verify that that the far-field behaviours, both for $t<0$ and $t>0$, are the same.

LHS and the second term on the RHS of (23), and takes the form

$$
h \sim\left(\frac{1}{2-a}\right) \frac{x^{1-a}}{t} .
$$

This is a collapsing solution where the surface tension gradients act to draw fluid away from the contact line. As was the case in problem considered in section 2 there is also a (largely irrelevant) exact steady solution where $s(t)=0$ and

$$
h=\sqrt{\frac{2}{a+1}} x^{(a+1) / 2} .
$$

\subsection{Behaviour after the halting time}

Since the analysis now undertaken is similar to that presented in sections 2.1 and 2.2, we shall omit some of the details. For $t>0$ the appropriate similarity 

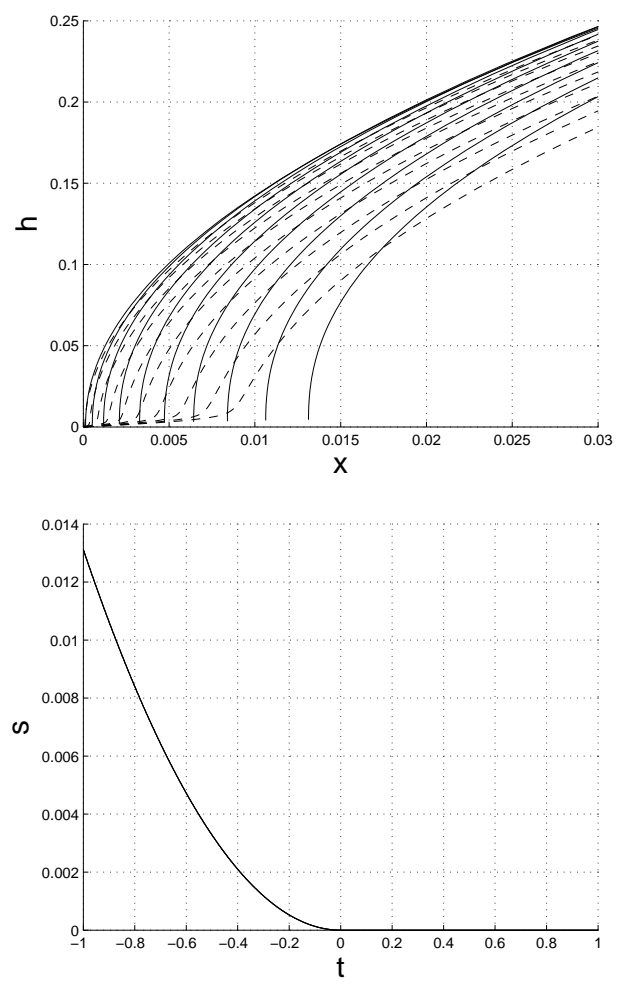

Figure 4: The left-hand panel shows the local solution to the PDE (9) plotted at 20 equally spaced times between -1 and 1 . Here, the solid and dashed curves show the evolution for $t<0$ and $t>0$ respectively. The right-hand panel shows the position of the contact line during this evolution.

reduction of $(23)$ is $s(t)=0$ and

$$
h(x, t)=t^{(a+1) /(1-3 a)} H(\phi) \quad \text { with } \quad \phi=x t^{2 /(3 a-1)} .
$$

Under this reduction the resulting ODE problem (i.e. the transformed version of (23) and its local boundary conditions) is

$$
\left(\frac{a+1}{1-3 a}\right) H+\left(\frac{2}{3 a-1}\right) H^{\prime} \phi=\left(H^{3} H^{\prime}-\phi^{a} H^{2}\right)^{\prime} \quad \text { with } \quad H(0)=0 .
$$

Near the contact line, where $\phi \rightarrow 0^{+}$, we find the following asymptotic behaviours of solutions to (29):

$$
\begin{array}{r}
H \sim\left(\frac{1}{2-a}\right) \phi^{1-a}+\frac{\left(4 a^{2}-7 a+3\right)}{5 a(a-2)^{3}} \phi^{2-4 a}+A_{1} \phi^{\left(4-5 a-a^{2}\right) /(4 a+2)} \\
+O\left(\phi^{3-7 a}\right) \quad \text { as } \phi \rightarrow 0^{+},
\end{array}
$$


where $A_{1}$ is an as yet undetermined constant ${ }^{1}$. This is a local solution which is dominated by surface tension gradients that pull fluid away from the contact line. In the far-field there is only one possible behaviour, namely

$$
H \sim A_{2} \phi^{(a+1) / 2}+(1+2 a)\left(\frac{A_{2}^{4}(a+1)}{2}-A_{2}^{2}\right) \phi^{2 a}+O\left(\phi^{(7 a-1) / 2}\right) \quad \text { as } \quad \phi \rightarrow+\infty .
$$

Here $A_{2}$ is an undetermined constant.

\subsection{Behaviour prior to the halting time}

When $t<0$ the similarity reduction and resulting ODE problem are

$$
\begin{aligned}
& h(x, t)=(-t)^{(a+1) /(1-3 a)} H(\phi), \quad \phi=x(-t)^{2 /(3 a-1)}, \quad s(t)=\Phi(-t)^{2 /(1-3 a)}, \\
& \left(\frac{a+1}{3 a-1}\right) H+\left(\frac{2}{1-3 a}\right) H^{\prime} \phi=\left(H^{3} H^{\prime}-(\phi-\Phi)^{a} H^{2}\right)^{\prime} \quad \text { with } \quad H(\Phi)=0
\end{aligned}
$$

Near the contact line where $\phi \rightarrow \Phi^{+}$the asymptotic behaviour of solutions to (33) is

$H \sim\left(\frac{6 \Phi}{1-3 a}\right)^{1 / 3}(\phi-\Phi)^{1 / 3}+\frac{6^{2 / 3}(1-3 a)^{1 / 3}}{2(3 a+4) \Phi^{1 / 3}}(\phi-\Phi)^{a+2 / 3}+o\left((\phi-\Phi)^{a+2 / 3}\right) \quad$ as $\quad \phi \rightarrow \Phi^{+}$

In the far-field there are two possible behaviours for solutions to (33). The first of which, $H \sim(3 / 10)^{1 / 3} \phi^{2 / 3}$ as $\phi \rightarrow+\infty$, may be discounted since it cannot match to the solution for $t>0$. The second behaviour, which will be retained, is:

$H \sim B \phi^{(a+1) / 2}+(1+2 a)\left(B^{2}-\frac{B^{4}(a+1)}{2}\right) \phi^{2 a}+O\left(\phi^{(7 a-1) / 2}\right) \quad$ as $\quad \phi \rightarrow+\infty$.

Here $B$ is an as yet undetermined constant.

\subsection{Results}

In the case when $\sigma_{x} \sim(x-s(t))^{a}$ as $h \rightarrow 0^{+}$a shooting scheme may be constructed in an analogous way to that described in section 2.3. Plots of the results for $a=1 / 10$ are shown in figure 5 . It was found that

$$
\Phi \approx 0.128, \quad B=A_{2} \approx 1.145, \quad \text { and } \quad \mathcal{A}_{2} \approx 11.348
$$

As with the case $\sigma_{x} \sim 1$ the numerical results strongly indicated that the above values of the parameters are unique. The variation of $\Phi$ with $a$ is shown in figure 6. Though the asymptotic analysis is valid for $-1 / 3<a<1 / 3$ the range of $a$ over which physically plausible results can be calculated appears to

\footnotetext{
${ }^{1}$ We note that in the behaviour (30), the ordering of the second, third and fourth terms depends on the value of $a$.
} 
be more limited. We were unable to find any numerical solutions for $a<0$. We conjecture that this is caused by the fact that in such cases the surface tension gradient is unbounded at the contact line. When $a$ exceeds a value of about 1/4 numerical solutions may still exist but $\Phi$ seems to be so small that it becomes increasingly difficult to solve the shooting problem reliably. We also note that for all $a>0$ solutions are qualitatively different from those examined in section 2 when $a=0$, for the "kinked" behaviour of the solutions for $t>0$ seen in figure 4 is absent. Finally, we note that $\Phi$ is not a monotonic function of $a$, and hence the deceleration of the $t<0$ wave is not monotonic, but has a maximum close to $a=1 / 10$.
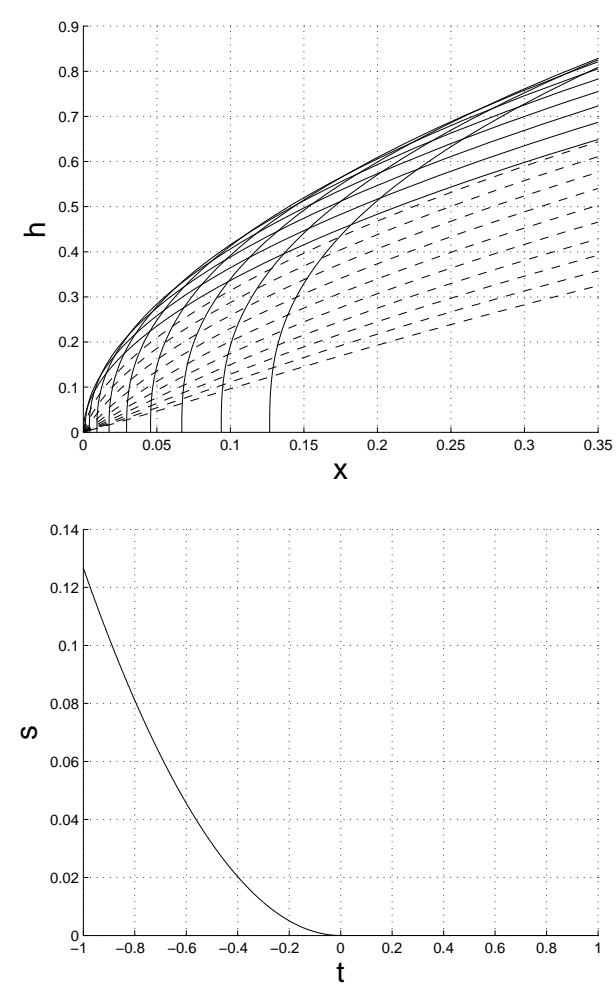

Figure 5: The left-hand panel shows the local solution to the PDE (23) (with $a=1 / 10$ ) plotted at 20 equally spaced times between -1 and 1 . The solid and dashed curves show the evolution for $t<0$ and $t>0$ respectively. The right-hand panel shows the position of the contact line during the evolution. 


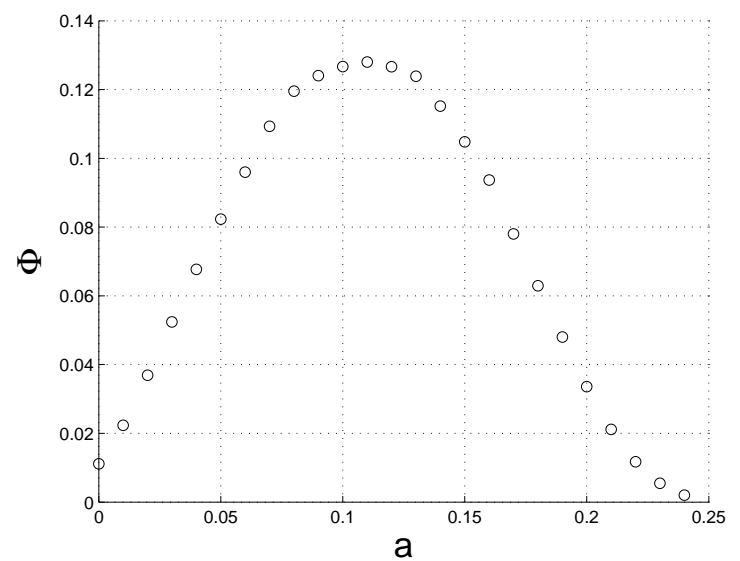

Figure 6: A plot of the variation of $\Phi$ with $a$.

\section{The case $\sigma_{x} \sim h^{b}$ as $h \rightarrow 0^{+}$}

We now examine a final case where the surface tension gradient depends on the local film height, i.e. $\sigma_{x} \sim h^{b}$ as $h \rightarrow 0^{+}$. Having removed superfluous coefficients by scaling, the local governing PDE and boundary conditions may be written as

$$
\frac{\partial h}{\partial t}=\frac{\partial}{\partial x}\left(h^{3} \frac{\partial h}{\partial x}-h^{b+2}\right), \quad h(s(t), t)=0, \quad \dot{s}=-h^{2} \frac{\partial h}{\partial x}+\left.h^{b+1}\right|_{x=s(t)} .
$$

We shall restrict our interest to the case

$$
-1<b<\frac{1}{2}
$$

Again, the lower bound ensures that surface tension gradients are sufficiently weak to allow an advancing wave, and the upper bound ensures that the surface tension gradients are sufficiently strong to halt the contact line at some finite time during the evolution. First, we examine travelling wave type solutions to the governing PDE. We find that there are two types of wave. The first is an advancing wave driven by gravity that takes the form

$$
h \sim(-3 \dot{s})^{1 / 3}(x-s(t))^{1 / 3},
$$

and arises due to a balance between the time derivative and the first term on the RHS of (37). The second behaviour arises due to a balance between the term on the LHS and the second term on the RHS of (37) and takes the form

$$
h \sim\left(\frac{1}{b+2}\right)^{1 /(b+1)} x^{1 /(b+1)} t^{-1 /(b+1)} .
$$


This is a collapsing solution driven by surface tension gradients. As with the previous cases we have examined there is also an (essentially irrelevant) exact steady solution

$$
h=(2-b)^{1 /(2-b)} x^{1 /(2-b)} .
$$

Since much of the ensuing analysis proceeds in a similar manner to that carried out in section 2, we shall suppress some of the details for brevity.

\subsection{Behaviour after the halting time}

For $t>0$ we have $s(t)=0$ and the similarity solution and governing ODE problem are

$$
\begin{array}{r}
h=t^{1 /(1-2 b)} H(\phi) \quad \text { with } \quad \phi=x t^{(2-b) /(2 b-1)}, \\
\left(\frac{1}{1-2 b}\right) H+\left(\frac{2-b}{2 b-1}\right) H^{\prime} \phi=\left(H^{3} H^{\prime}-H^{b+2}\right)^{\prime} \quad \text { with } \quad H(0)=0 .
\end{array}
$$

For $t>0$, near the contact line as $\phi \rightarrow 0^{+}$solutions to (43) have the following asymptotic behaviour:

$$
\begin{aligned}
H \sim\left(\frac{1}{b+2}\right)^{1 /(b+1)} \phi^{1 /(b+1)}+\left(A_{1}+\frac{(3-b)(2 b-1)}{(1+b)^{3}}\right. & \left.\left(\frac{1}{b+2}\right)^{4 /(b+1)} \log \phi\right) \phi^{2(1-b) /(1+b)} \\
& +o\left(\phi^{2(1-b) /(1+b)}\right) \text { as } \phi \rightarrow 0^{+} .
\end{aligned}
$$

Here $A_{1}$ is an as yet undetermined constant. This is a local solution that is largely governed by surface tension gradients which act to draw fluid away from the contact line. In the far-field solutions to (43) have behaviour

$$
\begin{aligned}
H \sim A_{2} \phi^{1 /(2-b)}+( & \left.\frac{(2+b)}{(2-b)^{2}} A_{2}^{4}-\frac{(2+b)}{(2-b)} A_{2}^{2+b}\right) \phi^{2 b /(2-b)} \\
& +O\left(\phi^{(1-4 b) /(b-2)}\right) \text { as } \phi \rightarrow+\infty
\end{aligned}
$$

where $A_{2}$ is an as yet undetermined constant.

\subsection{Behaviour prior to the halting time}

When $t<0$ the appropriate similarity reduction and resulting ODE problem are given by:

$$
\begin{array}{r}
h=(-t)^{1 /(1-2 b)} H(\phi), \quad \phi=x(-t)^{(2-b) /(2 b-1)}, \quad s(t)=\Phi(-t)^{(2-b) /(1-2 b)}, \\
\left(\frac{1}{2 b-1}\right) H+\left(\frac{2-b}{1-2 b}\right) H^{\prime} \phi=\left(H^{3} H^{\prime}-H^{b+2}\right)^{\prime} \quad \text { with } \quad H(\Phi)=0 .
\end{array}
$$


The asymptotic behaviour of solutions to $(47)$ as $\phi \rightarrow \Phi^{+}$is

$$
\begin{array}{r}
H \sim\left(\frac{3 \Phi(2-b)}{(1-2 b)}\right)^{1 / 3}(\phi-\Phi)^{1 / 3}+\frac{(1-2 b)}{(2-b)(4+b)}\left(\frac{3(2-b)}{1-2 b}\right)^{(b+2) / 3} \Phi^{(b-1) / 3}(\phi-\Phi)^{(b+2) / 3} \\
+O\left((\phi-\Phi)^{(2 b+3) / 3)}\right) \quad \text { as } \phi \rightarrow \Phi^{+} .
\end{array}
$$

This is an advancing solution driven by pressure gradients due to gravity. In the far-field there are two possible behaviours. The first, that cannot match in a suitable fashion to a solution for $t>0$, is given by $H \sim(3 / 10)^{1 / 3} \phi^{2 / 3}$ as $\phi \rightarrow+\infty$. The second behaviour is given by

$$
\begin{aligned}
H \sim B \phi^{1 /(2-b)}+ & \left(\frac{(2+b)}{(2-b)} B^{2+b}-\frac{(2+b)}{(2-b)^{2}} B^{4}\right) \phi^{2 b /(2-b)} \\
& +O\left(\phi^{(1-4 b) /(b-2)}\right) \quad \text { as } \quad \phi \rightarrow+\infty .
\end{aligned}
$$

Here $B$ is an as yet undetermined constant.

\subsection{Results}

In the case considered in this section, i.e. $\sigma_{x} \sim h^{b}$ as $h \rightarrow 0^{+}$, a shooting scheme may be constructed in an exactly analogous way to that described in section 2.3. Plots of the numerical results for $b=1 / 5$ are shown in figure 7 . It was found that

$$
\Phi \approx 0.117, \quad B=A_{2} \approx 1.165, \quad \text { and } \quad A_{1} \approx 82.573 .
$$

We performed the same numerical uniqueness tests that were used for the case $\sigma_{x} \sim 1$ and the evidence indicated that the above parameter values are unique. The dependence of $\Phi$ on $b$ is shown in figure 8. In an analogous manner to the case studied in section 3 we were only able to obtain solutions over a subset of the range $-1<b<1 / 2$. We conjecture that solutions are unavailable for $b<0$ because the surface tension gradient is unbounded at the contact line. When $b$ exceeds a value of about $2 / 5$ solutions may still exist but the shooting problem becomes too stiff to solve reliably. We also note that, like the solution shown in figure 5 , the solution for $b=1 / 5$ does not exhibit the "kink" feature that we observed when $\sigma_{x} \sim 1$. Lastly, we note that the maximal rate of deceleration of the contact line occurs when $b \approx 1 / 5$.

\section{$5 \quad$ Numerical experiments}

The purpose of this study is to explain the asymptotic structure of halting contact lines, and not to carry out detailed numerical calculations. It is nevertheless worth briefly reporting some numerical experiments that were undertaken (a) 

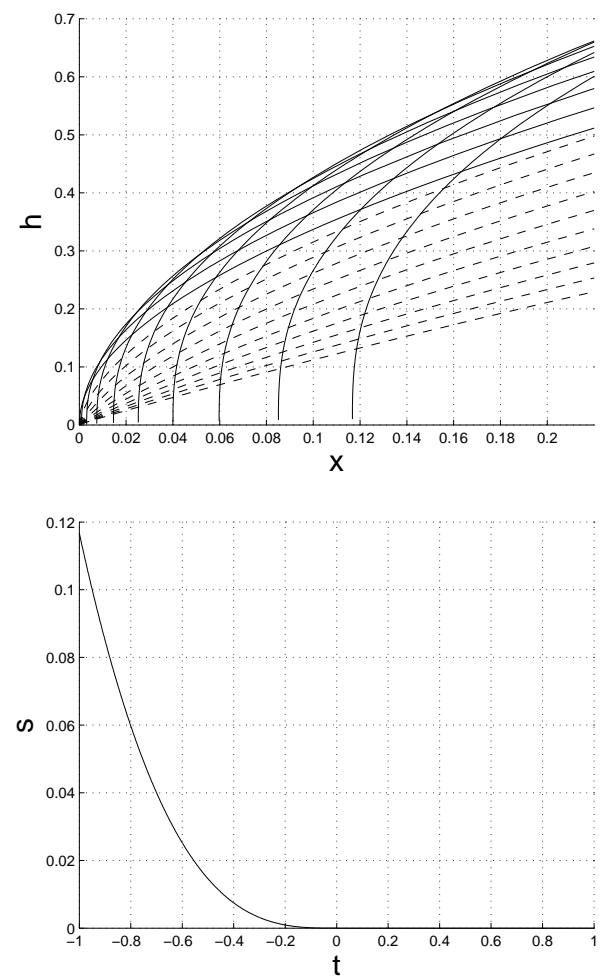

Figure 7: The left-hand panel shows the local solution to the PDE (37) (with $b=1 / 5$ ) plotted at 20 equally spaced times between -1 and 1 . The solid and dashed curves show the evolution for $t<0$ and $t>0$ respectively. The right-hand panel shows the position of the boundary during the evolution.

to try to help to understand the asymptotic results and (b) to suggest the plausibility of the results that have been generated.

The numerical experiments reported below were carried out using a first order central finite difference scheme. The complications associated with the moving boundary were avoided by only solving for the evolution after the contact line has halted, i.e. only for $t>0$. By doing so, one need only impose the condition $h(0, t)=0$ at the stationary contact line. For the case studied in section 2 the initial condition was taken to be $h(x, 0)=B^{1 / 2} x^{1 / 2}$, with $B \approx$ 1.403 (for cases where $a \neq 0$ or $b \neq 0$ the analogous solution was used) in order to be consistent with the similarity solution at $t=0$, see section 2.4. To close the problem we imposed the condition $h_{x}(X, t)=0$, for some positive constant $X$. We note that although this boundary condition is inconsistent with the initial data we have numerically verified that for sufficiently large $X$ the solution near the contact line is unaffected by the choice of $X$.

Plots of a selection of the results of the numerical experiments are shown 


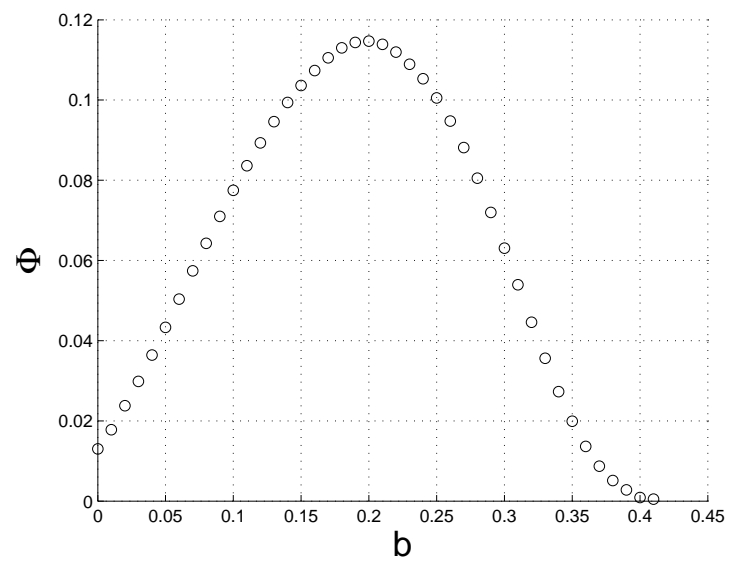

Figure 8: A plot of the variation of $\Phi$ with $b$.

in figures 9, 10 and 11. The qualitative agreement between the self-similar solution in figure 4 and the numerical solution in figure 9 is clear. Assessing the agreement between 5 and 10, and, 7 and 11 is more difficult, primarily because there is no "kink" feature to suggest appropriate length and time scales. Whilst these results are in themselves interesting and support the asymptotic results it is clear that a detailed numerical study may be more revealing.

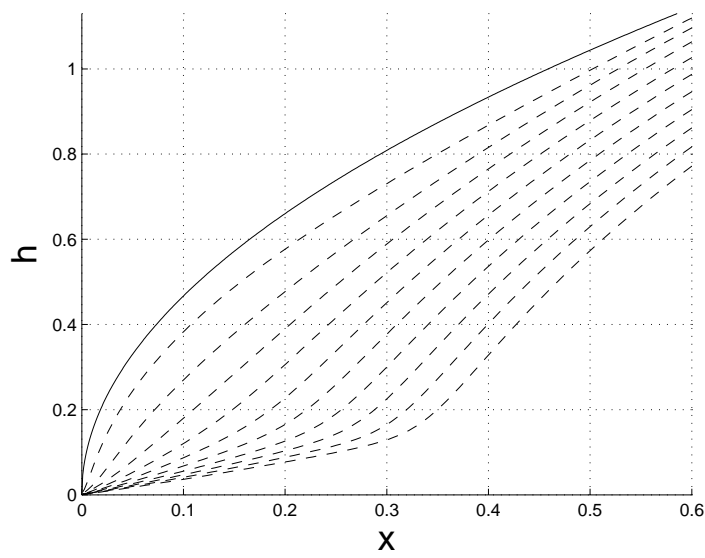

Figure 9: A plot of a wholly numerical solution of (9). The solid curve shows the initial condition and the dashed curves show the subsequent evolution. 


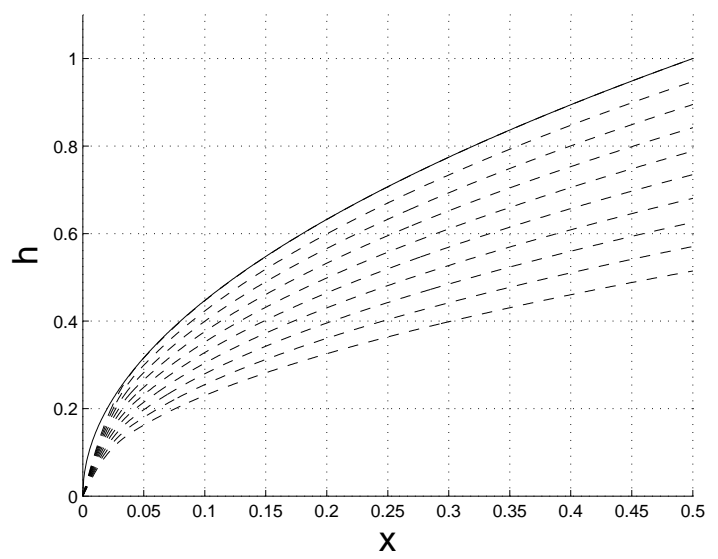

Figure 10: A plot of a wholly numerical solution of (23) with $a=1 / 10$. The solid curve shows the initial condition and the dashed curves show the subsequent evolution.

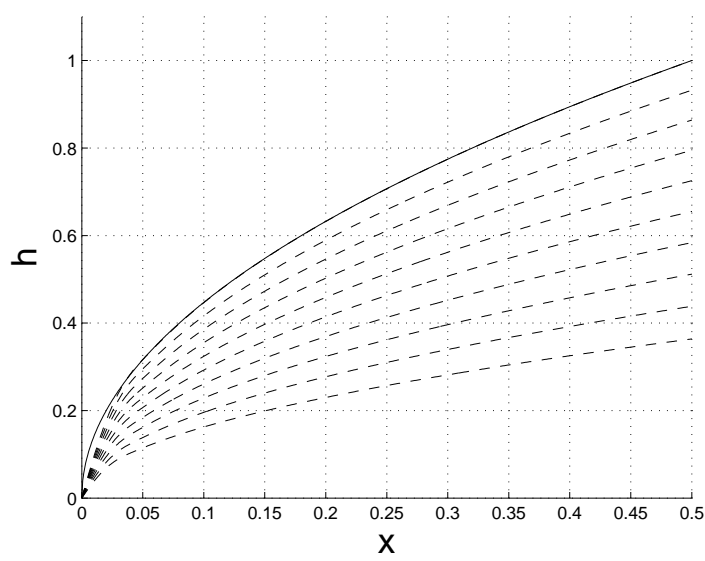

Figure 11: A plot of a wholly numerical solution of (37) with $b=1 / 5$. The solid curve shows the initial condition and the dashed curves show the subsequent evolution.

\section{Discussion and conclusions}

In the sections above we have described the detailed asymptotic structure of solutions where an evolving contact line driven by gravity and surface tension gradients halts in finite time (at a finite position) owing to the surface tension gradients. The stopping is driven by local effects alone, and the solutions examined may or may not be unique. Though Gandarias'[28] Lie group analysis 
of the classical point symmetries of (6) (which we have independently verified) shows that the similarity reductions used above are essentially the only nontrivial ways in which (6) may be reduced to an ODE, it is nevertheless possible that there may be other types of evolution that are controlled by global effects (for example, locally driven "waiting time" solutions to a related problem [10] were later found to be sub-cases of a more complicated solution structure, see Lacey et. al.[9]).

The effect of three different types of surface tension were examined in sections 2,3 and 4 respectively. It appears however that the case $a=b=0$ is special, in some sense, as the solution that it produces after the halting time displays a characteristic "kinked" behaviour where the film appears to want to try to reverse (like the absorption case covered in another study[17]) but cannot as it is essentially "pinned" at $x=0$. We speculate that this behaviour is unique to the case $a=b=0$ as this is the only instance that we have studied where $\sigma_{x}$ is finite (rather than zero or infinite) at the contact line.

One intriguing possibility that is suggested by the cases that have been examined is that the halting effect could be exploited to control drop spreading, i.e. by heating the substrate in a particular manner. In this way (for example) an industrial process could be designed that produces drops that spread to a desired (and carefully controllable) size. To make this work it would be necessary know exactly how both the stopping time and position depend on the initial conditions. Unfortunately the lack of a natural time scale suggests that it will be very hard to analyse the halting time and position for arbitrary initial value problems, and it may be that a purely numerical attack is the only practical

option. Though such a detailed numerical study is outside the scope of the current paper, there are other reasons for carrying one out. For example, it would be of great interest to understand the details of how $s(t)$ changes with $a$ and $b$, what happens when $a$ and $b$ are small and negative, and whether or not halting solutions can exist when $a$ and $b$ are either close to the limits of or completely outside the ranges given by (24) and (38).

Finally, it must be reiterated that the numerical shooting method outlined in section 2.3 and later used to calculate detailed solutions is not rigorous. Though the numerical calculations involved have been carried out with as much care as possible, the current lack of appropriate rigorous results and existence and uniqueness theorems remains a concern.

\section{References}

[1] J. Thomson. On certain curious motions observable on the surfaces of wine and other alcoholic liquers. Phil. Mag., 10(67):330-333, 1855.

[2] J. R. A. Pearson. On convective cells induced by surface tension. J. Fluid Mech., 4:489-500, 1958.

[3] A. Oron, S. H. Davis \& S. G. Bankoff. Long-scale evolution of thin liquid films. Rev. Mod. Phys., 69:931-980, 1997. 
[4] E. D. Hondros, M. McLean \& K. C. Mills, Marangoni and interfacial phenomena in materials processing: originating from contributions to a discussion of the Royal Society of London. IOM Communications Limited, 1998.

[5] M. G. Velarde \& R. K. Zeytourian, Interfacial phenomena and the Marangoni effect. Springer-Verlag Wien New York, 2002.

[6] T. G. Myers. Thin films with high surface tension. SIAM review, 40(3):441462, 1998.

[7] H. E. Huppert. The propogation of two-dimensional and axisymmetric viscous gravity currents over a rigid horizontal substrate. Journal of Fluid Mechanics, 121:43-58, 1982.

[8] D. G. Aronson, L. C. Caffarelli \& S. Kamin. How an initially stationary interface begins to move in porous medium flow. SIAM Journal of Mathematical Analysis, 14:639-658, 1983.

[9] A. A. Lacey. Initial motion of the free boundary for a non-linear diffusion equation. IMA J. Appl. Math., 31:113-119, 1983.

[10] A. A. Lacey, J. R. Ockendon \& A. B. Tayler. "Waiting time" solutions of a nonlinear diffusion equation. SIAM J. Appl. Math., 42:1252-1264, 1982.

[11] V. A. Galaktionov, S. I. Shmarev \& J. L. Vazquez. Regularity of interfaces in diffusion processes under the influence of strong absorption. Archive for Rational Mechanics and Analysis, 149:183-212, 1999.

[12] M. A. Herrero \& J. L. Vazquez. The one-dimensional nonlinear heat equation with absorption: regularity of solutions and interfaces. SIAM Journal of Mathematical Analysis, 18:149-167, 1987.

[13] S. Kamin \& L. Veron. Existence and uniqueness of the very singular solution of the porous medium equation with absorption. Journal d'Analyse Mathematique, 51(1):245-258, 1988.

[14] B. F. Knerr. The behaviour of the support of solutions of the equation of nonlinear heat conduction with absorption in one dimension. Transactions of the American Mathematical Society, 249:409-424, 1979.

[15] R. Ferreira \& J. L. Vazquez. Extinction behaviour for a fast diffusion equations with absorption. Nonlinear analysis, 43:943-985, 2001.

[16] R. E. Grundy. The asymptotics of extinction in nonlinear diffusion reaction equations. Journal of the Australian Mathematical Society, 33:413-428, 1992.

[17] J. M. Foster, C. P. Please, A. D. Fitt \& G. Richardson. The reversing of interfaces in slow diffusion processes with strong absoprtion. SIAM J. Appl. Math., 72(1):144-162, 2012. 
[18] S. J. Chapman, P. H. Trinh \& T. P. Witelski. Exponential asymptotics for thin film rupture. SIAM Journal on Applied Mathematics, 73(1):232-253, 2013.

[19] J. A. Diez \& L. Kondic. On the breakup of fluids films of finite extent Physics of Fluids, 19:072107, 2007.

[20] D. Vaynblat, J. R. Lister \& T. P. Witelski. Rupture of thin viscous films by van der Waals forces: Evolution and self-similarity. Phil. Mag., 13(5):11301140, 2001.

[21] W. W. Zhang \& J. R. Lister. Similarity solutions for van der Waals rupture of a thin film on a solid substrate. Physics of Fluids, 11(9):2454-2462, 1999.

[22] Y. M. Yatim, B. R. Duffy, S. K. Wilson \& R. Hunt. Similarity solutions for unsteady gravity-driven slender rivulets. QJMAM, 64(4):455-480, 2011.

[23] Y. M. Yatim, B. R. Duffy \& S. K. Wilson. Travelling-wave similarity solutions for a steadily translating slender dry patch in a thin fluid film. Physics of Fluids, 25:052103, 2013.

[24] K. Mikula. Numerical solution of nonlinear diffusion with finite extinction phenomenon. Acta Mathematica Universitatis Comenianae, 64(2):173-184, 1995.

[25] T. Nakaki. Numerical interfaces in nonlinear diffusion equations with finite extinction phenomena. Hiroshima Mathematical Journal, 18:373-397, 1988.

[26] T. Nakaki \& K. Tomoeda. A finite difference scheme for some nonlinear diffusion equations in an absorbing medium: support splitting phenomena. SIAM J. Numerical Analysis, 40(3):945-964, 2002.

[27] S. D. Howison, J. A. Moriarty, J. R. Ockendon, E. L. Terrill \& S. K. Wilson. A mathematical model for drying paint layers. J. Eng. Math., 32:377-394, 1997.

[28] M. L. Gandarias. Classical point symmetries of a porous medium equation. J. Phys. A: Math. Gen., 29:607-633, 1996.

[29] E. O. Tuck \& W. Schwartz. A numerical and asymptotic study of some third-order ordinary differential equations relevant to draining and coating flows. SIAM Review, 32(3):453-469, 1990.

[30] L. F. Shampine \& M. W. Reichelt. The MATLAB ODE suite. SIAM Journal on Scientific Computing, 18:1-22, 1997. 